\title{
Sources of evidence
}

\author{
Jeyanthi John \\ Senior Researcher, Centre for Evidence-based Dentistry, Oxford UK
}

\begin{abstract}
In the last issue we looked at levels of evidence. ${ }^{1}$ In this article we take an overview of the sources of this evidence in this information age. In later articles we will then look in more depth at some of these sources themselves.

Evidence-Based Dentistry (2003) 4, 37-39. doi:10.1038/sj.ebd.6400186
\end{abstract}

\section{The information explosion}

There is now a drive for all health professionals, including dentists, to practise evidence-based healthcare. This requires all clinical decisions to be underpinned by up-to-date information about the best available options for each situation. Clinicians therefore need to keep abreast of the rapid advances in dentistry (a daunting task for most busy practitioners), and to constantly evaluate their clinical practice against this evolving information base. To further add to this, the Internet has resulted in an explosion in the amount of information available to patients. Clinicians will be familiar with the patient who arrives armed with information downloaded from the WWW about the "latest" treatments that have been "proved" to be the best for anyone with his/her condition! In this climate it is important for all members of the dental team to be able to advise their patients about the most appropriate options and be able to back up their advice with the available evidence.

\section{Evidence-based dentistry}

The American Dental Association (ADA) defines evidence-based dentistry (EBD) as, "an approach to oral health care that requires the judicious integration of systematic assessments of clinically relevant scientific evidence, relating to the patient's oral and medical condition and history, with the dentist's clinical expertise and the patient's treatment needs and preferences". This definition of EBD includes the use of research evidence (which extends beyond any individual clinician's personal experience) but does not focus solely on it. It acknowledges the importance of clinical skills and judgement in assessing the various options in relation to individual patient's risks and benefits, as well as involving the patient's own preferences regarding the type of care they would like to receive (Figure 1). This process involves several stages: ${ }^{2}$

\section{Levels of evidence}

Evidence is the product of well-designed and well-conducted research investigations. Good research contributes to the body of knowledge which evolves constantly with the addition of new information. Appraising this evidence can then inform clinical decisions. In an earlier article $^{1}$ the levels of evidence developed by the Centre for Evidence-based Medicine were outlined, a simplified classification of which is shown below: ${ }^{2}$

- Strong evidence from at least one published systematic review of multiple, welldesigned randomised controlled trials (RCT) - Strong evidence from at least one published properly-designed, RCT of appropriate size and in an appropriate clinical setting

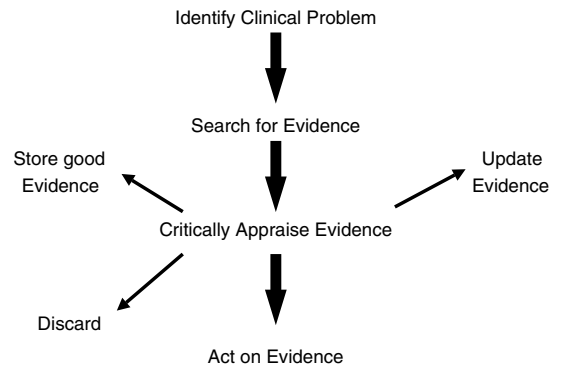

Figure 1. The evidence-based dentistry process.
- Evidence from published, well-designed trials without randomisation, single-group, pre- post-, cohort, time-series or matched case-controlled studies

- Evidence from well-designed, experimental studies from more than one centre or research group

- Opinions of respected authorities, based on clinical evidence, descriptive studies or reports of expert consensus committees.

\section{Sources of evidence}

Evidence is available from a wide range of sources. Each of them has advantages and disadvantages. Whatever the sources, if we are to be evidence-based, we should remember that the evidence we find should be appraised critically before we apply it to the patient.

Colleagues This is usually the first option for most of us. Many healthcare professionals, not just dental team members, will remember learning to carry out various procedures 'on-the-job' ie, from senior colleagues or from a peer who has had experience of the procedure.

An advantage of this source of evidence is that, chances are, one of our colleagues will have an answer, saving us spending time delving through books or papers. A survey of 300 general dental practitioners in the north-west of England found that $60 \%$ of respondents turned to friends and colleagues for help and advice when faced with clinical uncertainties. ${ }^{3}$ There is also the option of contacting a specialist in the field who can provide an expert opinion. Alternatively, the expert can point us in the direction of a good paper from which to begin our search for an answer to our clinical problem.

Disadvantages of this source are that although the above definition of EBD acknowledges the value of expert knowledge and experience, these opinions are of the lowest level and should be considered only in relation to other types of evidence. Experts often disagree, may not 
be up-to-date in their knowledge of the particular issue you are raising, or may simply disagree with the current evidence, always sticking to their own preferred mode of treatment.

Books Books make handy references and most of us have a good selection dating back to dental school days. Books are a good source of comprehensive established information. The information is laid out in well-defined sections with an index of terms making them useful as quick references for basic background information. The disadvantage is that, due the extended time it takes to research, script and publish a book (sometimes years), some of the information becomes out-of-date quickly. Books are unreliable as a major source of current information.

The Internet The Internet has revolutionised the way most people work or, indeed, spend their leisure time. The National Statistics Omnibus Survey from July 2001 indicates that $51 \%$ of adults in Great Britain have accessed the Internet at some point (www.e-envoy.gov.uk/ ukonline/progress/anrep). Between April and June 2001, 38\% of all UK households could access the Internet from home. These proportions will grow with the continuing government support aimed at ensuring that, "everyone in the UK who wants it will have access to the Internet by 2005" (www.statistics.gov.uk/ pdfdir/int0901.pdf). The Internet has also made the practice of EBD possible. Dental team members can access information from any number of sources electronically.

Information on practically any subject can therefore be obtained relatively quickly and easily from any computer with access to the Internet. Many organisations and journals maintain an Internet presence (see below) that is freely available to everyone. Research evidence, clinical guidelines, patient information and resources can all be obtained by anyone with a computer that has access to the Internet.

Disadvantages are that there is a enormous amount of information available through the WWW and finding the information you need can be tedious, frustrating and time-consuming. At the time of writing, using the search term "evidence based dentistry" in the WWW search engine 'Google' (www.google.com), resulted in a list of 116000 sites. The information is usually not subject to any form of review and it is difficult to differentiate the facts from other useless or inaccurate information. The danger here is that patients may believe some of the erroneous information they obtain from the Internet. Many organisations have published criteria to help individuals assess the quality of health-related websites but there is no standard set of guidelines. ${ }^{4}$

Journals Reading journals is obviously one method of keeping track of the latest advances in your field of interest. There is a range of journals in both general dentistry and in the various specialties.

Journals contain much more up-todate information than books because articles are generally published within months of submission. Many journals now have a WWW presence which enables subscribers to obtain access to an article, a particular issue or subscribe to the electronic edition of the journal. Some journals provide an "e-mail alert" facility that enables readers to receive the contents lists of the journals in their electronic mailbox, allowing them to scan and retrieve relevant titles regularly.

Nevertheless, there are over 900 dental journals available world-wide including generalist publications, eg, the British Dental Journal and Journal of the American Dental Association, and specialist journals, eg, Journal of Periodontology or the European Journal of Orthodontics. One study that quantified high-quality prosthetic dental clinical trials indexed on Medline between 1990 and 1999 identified 10258 articles published in English. ${ }^{5}$ These were published in more than 60 different journals: approximately $50 \%$ of the articles were published in 14 journals, whereas the remaining articles were published in 46 journals. With this quantity increasing all the time, staying abreast of developments, even in one's own particular area of work, becomes a monumental task.

A strategy to keep up with relevant research articles in journals is outlined by Sackett et al, $^{6}$ derived from an article by Haynes et al.: ${ }^{7}$

Decide which of the high circulation generalist and specialist journals have most articles relevant to your clinical practice. Review current contents regularly and read as many of these as you can.

Collaborate with friends and colleagues to develop and circulate complementary subscriptions.
Another option is to regularly use an evidence-based supplement, such as this one. This supplement provides an overview of a selection of high-quality research articles relating to a wide range of clinical practice. This is particularly useful for general dental practitioners, who need to keep abreast of developments in dentistry as a whole, and for specialists, who are keen to remain aware of developments in other areas of dentistry.

Electronic databases A range of specialised bibliographic databases are available electronically, each focusing on a particular speciality. For example, MEDLINE indexes a wide range of dental (and medical) journals. Other databases include CINAHL (Cumulative Index of Allied Health Literature) which indexes nursing journals and Psychinfo which indexes books and journals focusing on the behavioural sciences and mental health. Medline is created and maintained by the US National Library of Medicine of the National Institutes of Health. It contains over 11 million references, of which about $76 \%$ include English language abstracts from over 4300 journals published in more than 70 countries. Over 400000 new references are added per year, at a rate of nearly 8000 publications per week. About 320 of the over 900 dental journals are indexed on this database. MEDLINE can be accessed through a variety of ways through the Internet including PubMed (www.ncbi.nlm.nih.gov/PubMed).

The chief advantage is that users can search a number of journals for articles in a particular area using words and phrases. Abstracts are available for about a third of the articles, which can help the reader identify those that might be useful. PubMed also includes a "related articles" link next to every reference which directs readers to similar articles. In some cases there are direct links to the original article: in the case of the Canadian Dental Journal this links directly to the full text of the article.

Not all relevant references, however, are indexed on an electronic database. It is useful to review the bibliographies of papers identified in electronic searches to improve the yield of articles.

Specialist organisations There are some organisations dedicated to producing high-quality evidence to inform healthcare. Three of these are highlighted here. First, the Cochrane Colla- 
boration (www.cochrane.org) is an international network that maintains an electronic database of systematic reviews, meta-analyses and randomised controlled trials. The organisation has several Cochrane Review Groups each of which focuses on a particular topic. The Cochrane Oral Health Group (www.cochrane-oral.man.ac.uk/) is based at the University of Manchester. They carry out systematic reviews in oral health and maintain a register of RCT compiled using a combination of electronic and hand searches.

The NHS Centre for Reviews and Dissemination (CRD) is based in York, UK (www.york.ac.uk/inst/crd) and is a sibling organisation of the UK Cochrane Centre within the NHS Research and Development Programme. It undertakes and commissions reviews of researchfindings on the effectiveness of healthcare relevant to the UK National Health Service (NHS). CRD maintains databases of abstracts of good-quality reviews of health research, abstracts of economic evaluations of health and health technology assessments. It also provides an information and enquiry service on reviews and economic evaluations for healthcare professionals, purchasers and providers, NHS managers, information providers, health service researchers and consumer organisations.

The National Library for Health (NeLH; www.nelh.nhs.uk) provides a digital library for healthcare professionals to aid healthcare-related decisions. It provides access to a number of resources such as Medline and the British National Formulary. An oral health branch of the $\mathrm{NeLH}$ has just been launched (www.nelh.nhs.uk/oralhealth) and provides current evidence for oral healthcare decisions.

The advantage of these organisations is that they use stringent criteria to review information from the research available on a particular topic and they provide a 'bottom-line' on the subject. The criteria used are always stated up-front making it possible for the reader to form an independent judgement of the results.
There is not always sufficient information to answer a particular question, however. Sometimes the bottom-line is that there is no bottom-line.

Guidelines Clinical practice guidelines have been defined as, "systematically developed statements to assist practitioners and patients in arriving at decisions on appropriate health care for specific clinical circumstances" ${ }^{8}$ They are not designed to replace clinical experience and knowledge but are recommendations to assist healthcare professionals in clinical practice.

The National Institute of Clinical Excellence (NICE) in the UK produces clinical guidelines for the management of specific disease conditions within the NHS (England and Wales). The guidelines are produced in response to requests from the Department of Health and the National Assembly for Wales. All NICE guidelines are based on the best available evidence and involve all stakeholders, eg, healthcare professionals and patient/ carer organisations.

These evidence-based guidelines synthesise the evidence and provide a guide to the optimum treatment option(s), saving practitioner-time. NICE also produces patient versions, which are designed to help patients make informed choices about their care. This can help facilitate the discussion between the clinician and the patient. The disadvantage of the guidelines is that they are general by their very nature, and may not be appropriate for every individual patient. This is where clinical experience and judgement becomes invaluable.

\section{Be aware of publication bias!}

Publication bias is, "any influence that reduces the amount of good science appearing in the literature". ${ }^{9}$ Publication bias can be of various types. Bias can result in studies with positive results getting published more often or sooner than those with negative or equivocal results. ${ }^{10}$ English-language articles are also more likely to be published than those of other languages. ${ }^{10}$ Publication bias results in a body of evidence skewed falsely in a particular direction. Interventions may also be tested unnecessarily because of the failure to publish findings from research that has already been conducted. It is therefore important for researchers to ensure that their work is published speedily after the completion of the study. It is also important for practitioners to be aware of this problem when investigating treatment options.

\section{Conclusions}

A wealth of resources exists to help dental team members practise EBD and keep up to date. As can be seen in this brief overview, a wide range of sources of differing quality is available. Each of these sources can be used to practise EBD but, in order to do this, dental team members need to acquire the appropriate skills to be able to identify and appraise the evidence and thereby provide the best care for their patients. There are courses available that teach these skills (see forthcoming events page) and we aim to provide some of the information to assist the practitioner in this journal.

1. Richards D. Not all evidence is created equal - so what is good evidence? Evidence Based Dentistry 2003; 4:17-18.

2. Richards $D$, Lawrence $A$. Evidence-Based Dentistry. Br Dentil J 1995; 179:270-273.

3. Iqbal A, Glenny AM. General dental practitioners' knowledge of and attitudes towards evidence based practice. Br Dent I 2002; 193:587-591.

4. Kim P, Eng TR, Deering MJ, Maxfield A. Published criteria for evaluating health related web sites: review. Br Med J 1999; 318:647-649.

5. Nishimura K, Rasool F, Ferguson MB, Sobel M, Niederman R. Benchmarking the clinical prosthetic dental literature on MEDLINE. J Prosthet Dent 2002; 88:533-541.

6. Sackett DLS, Haynes RB, Guyatt GH, Tugwell P. Clinical Epidemiology, a Basic Science for Clinical Medicine. Boston: Little, Brown: 2003.

7. Haynes RB, McKibbon KA, Fitzgerald D, Guyatt GH, Walker CJ, Sackett DL. How to keep up with the medical literature: II. Deciding which journals to read regularly. Ann Intern Med 1986; 105:309_ 312

8. Institute of Medicine. Clinical Practice Guidelines: Directions for a New Program. Washington, DC: National Academy Press;.

9. Chalmers T, Frank C, Reitman D. Minimising the three stages of publication bias. J Am Med Assoc 2003; 263:1392-1395.

10. Scholey JM, Harrison JE. Publication bias: raising awareness of a potential problem in dental research. Br Dent J 2003; 194:235-237. 